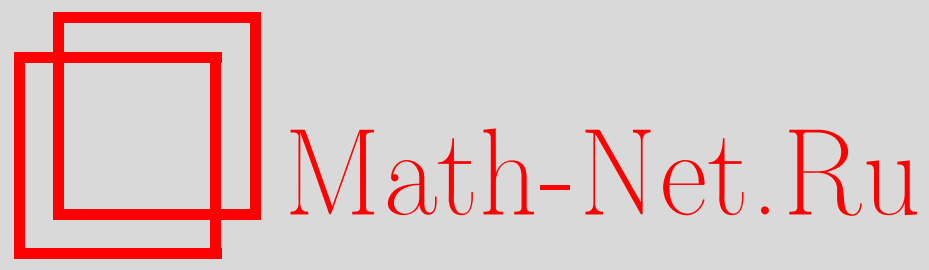

И. М. Кричевер, Алгебро-геометрические nортогональные криволинейные системы координат и решения уравнений ассоциативности, Функи. анализ и его прил., 1997, том 31, выпуск $1,32-50$

DOI: https://doi.org/10.4213/faa444

Использование Общероссийского математического портала Math$\mathrm{Net.Ru}$ подразумевает, что вы прочитали и согласны с пользовательским соглашением

http://www . mathnet.ru/rus/agreement

Параметры загрузки:

IP : 54.237 .206 .68

26 апреля 2023 г., 11:11:53

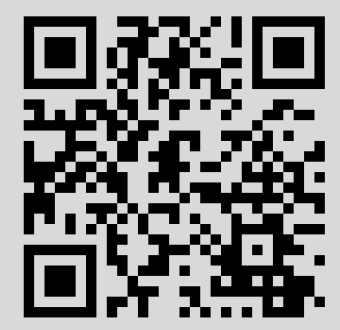


Функииональный анализ и его приложения

1997, т. 31, вып. 1, с. 32-50

УДК 517.9

\title{
Алгебро-геометрические $\boldsymbol{n}$-ортогональные криволинейные системы координат и решения уравнений ассоциативности
}

\author{
(C) 1997. И. М. КРИЧЕВЕР
}

\section{§1. Введение}

Более века, начиная со знаменитой работы Дюпена и Бинэ, опубликованной в 1810 г., проблема построения $n$-ортогональных криволинейных систем координат или плоских диагональных метрик

$$
d s^{2}=\sum_{i=1}^{n} H_{i}^{2}(u)\left(d u^{i}\right)^{2}, \quad u=\left(u^{1}, \ldots, u^{n}\right),
$$

относилась к числу важнейших проблем дифференциальной геометрии. Как задача классификации она была в основном решена в начале этого века. Решаюший вклад в богатую историю этой проблемы внес Дарбу в своей монографии [1].

Современный интерес к этой классической проблеме возник в начале 80-х годов, когда обнаружились ее глубокие связи с теорией интегрируемых квазилинейных $(1+1)$-мерных систем гидродинамического типа [2-4]. Теория таких систем была построена в работах Дубровина и Новикова как гамильтонова теория усредненных уравнений (уравнений Уизема) для периодических решений интегрируемых одномерных эволюционных уравнений теории солитонов. Позже в работе [5] было отмечено, что проблема классификации так называемых егоровских метрик, т.е. плоских диагональных метрик, таких, что

$$
\partial_{j} H_{i}^{2}=\partial_{i} H_{j}^{2}, \quad \partial_{i}=\partial / \partial u^{i},
$$

эквивалентна проблеме классификации массивных топологических моделей теории поля. Заметим, что условие (1.2) означает, что существует функция $\Phi(u)$, называемая потенциалом соответствующей метрики, такая, что $H_{i}^{2}(u)=\partial_{i} \Phi(u)$. Следует подчеркнуть, что «классические» результаты в теории $n$-ортогональных криволинейных систем координат носили в основном классификационный характер. Было показано, что локально общее решение уравнений Ламе

$$
\begin{gathered}
\partial_{k} \beta_{i j}=\beta_{i k} \beta_{k j}, \quad i \neq j \neq k, \\
\partial_{i} \beta_{i j}+\partial_{j} \beta_{j i}+\sum_{m \neq i, j} \beta_{m i} \beta_{m j}=0, \quad i \neq j,
\end{gathered}
$$

для так называемых коэффищиентов вращения

$$
\beta_{i j}=\partial_{i} H_{j} / H_{i}, \quad i \neq j,
$$


зависит от $n(n-1) / 2$ произвольных функций двух переменных. Уравнения $(1.3)$, (1.4) эквивалентны условиям зануления всех, априори ненулевых, коэффициентов тензора кривизны. (Уравнения (1.3) эквивалентны равенствам $R_{i j, i k}=0$, а уравнения (1.4) означают, что равны нулю остальные коэффициенты $R_{i j, i j}=0$.)

Если решение уравнений (1.3), (1.4) найдено, то соответствующие коэффициенты Ламе $H_{i}$ могут быть получены как решения системы линейных уравнений (1.5), совместность которых эквивалентна (1.3). Они зависят от $n$ произвольных функций одной переменной, которые являются данными Коши для системы (1.5),

$$
f_{i}\left(u^{i}\right)=H_{i}\left(0, \ldots, 0, u^{i}, 0, \ldots, 0\right) .
$$

После этого плоские координаты $x^{k}(u)$ можно найти из системы линейных уравнений

$$
\begin{aligned}
\partial_{i j}^{2} x^{k} & =\Gamma_{i j}^{i} \partial_{i} x^{k}+\Gamma_{j i}^{j} \partial_{j} x^{k}, \\
\partial_{i i}^{2} x^{k} & =\sum_{j=1}^{n} \Gamma_{i i}^{j} \partial_{j} x^{k},
\end{aligned}
$$

где $\Gamma_{i j}^{k}$ - коэффищиенты Кристоффеля метрики (1.1):

$$
\Gamma_{i k}^{i}=\partial_{k} H_{i} / H_{i}, \quad \Gamma_{i i}^{j}=-H_{i} \partial_{i} H_{i} / H_{j}^{2}, \quad i \neq j .
$$

Изложенная схема недостаточно эффективна для явного построения $n$-ортогональных криволинейных систем координат. Как следствие список явных примеров таких координат сравнительно невелик. Недавно он значительно пополнился за счет примеров, полученных в рамках теории Уизема. Например, в работе автора [6] было показано, что пространства модулей алгебраических кривых с фиксированными ростками локальных координат в отмеченных точках порождают плоские диагональные метрики.

В самое последнее время в работе Захарова [7] было обнаружено, что широкий класс решений уравнений (1.3) и (1.4) может быть получен в рамках процедуры «одевания», широко известной в теории интегрируемых солитонных уравнений. Уравнения (1.3) эквивалентны условиям совместности для системы вспомогательных линейных уравнений $\partial_{i} \Psi_{j}=\beta_{i j} \Psi_{i}, i \neq k$. Следовательно, любая известная в рамках метода обратной задачи схема может быть сравнительно легко применена для построения точных решений уравнений (1.3). Это может быть процедура одевания или, например, алгебро-геометрические конструкции теории конечнозонных решений нелинейных уравнений. При таком подходе решающим является выделение среди построенных решений тех, которые удовлетворяют соотношениям (1.4). Как было замечено в [8], метод дифференииальной редукиии, предложенный в [7] для решения последней задачи в рамках метода одевания, допускает естественную интерпретацию в терминах так называемой $\bar{\partial}$-проблемы. 
Основной целью настоящей работы является не столько построение конечнозонных или алгебро-геометрических решений уравнений Ламе, как разработка схемы, в рамках которой можно одновременно решить всю систему уравнений (1.3)-(1.9), т.е. получить вместе с коэффициентами Ламе и плоские координаты $x^{i}(u)$.

На первый взгляд предлагаемый подход к построению плоских диагональных метрик никак не связан с идеями конструкций из [7] и [8]. Мы рассматриваем базисные многоточечные функции Бейкера-Ахиезера $\psi(u, Q)$, однозначно определяемые их аналитическими свойствами на вспомогательных римановых поверхностях $\Gamma, Q \in \Gamma$, и непосредственно доказываем (без какого бы то ни было использования дифференциальных уравнений!), что при определенных ограничениях на исходные алгебро-геометрические данные значения $x^{k}(u)=\psi\left(u, Q_{k}\right)$ функции $\psi$ в наборе отмеченных точек на $\Gamma$ удовлетворяют уравнениям

$$
\sum_{k, l} \eta_{k l} \partial_{i} x^{k}(u) \partial_{j} x^{l}(u)=H_{i}^{2}(u) \delta_{i j}
$$

где $\eta_{k l}$ - постоянная матрица. Эти уравнения означают, что $x^{k}(u)$ являются плоскими координатами для диагональной метрики (1.1) с коэффициентами $H_{i}^{2}(u)$. При этом оказывается, что с точностью до постоянного множителя коэффициенты Ламе $H_{i}(u)$ равны главным членам разложения той же самой функции $\psi$ в точках $P_{i}$ на $\Gamma$, где $\psi$ имеет существенные особенности экспоненциального типа. Следует отметить, что ограничения на алгебро-геометрические данные, приводящие к (1.10), являются обобщениями ограничений, предложенных в работе [15] для описания потенциальных интегрируемых двумерных операторов Шрёдингера (см. также [16]).

В третьем параграфе работы мы связываем нашу конструкцию с подходом работ $[7,8]$ и доказываем, что функция Бейкера-Ахиезера $\psi$ является производящей функцией,

$$
\partial_{i} \psi(u, Q)=h_{i}(u) \Psi_{i}^{0}(u, Q), \quad H_{i}=\varepsilon_{i} h_{i}(u), \quad \varepsilon_{i}=\mathrm{const},
$$

для решений системы линейных уравнений

$$
\partial_{i} \Psi_{j}^{0}=\beta_{j i} \Psi_{i}^{0}, \quad \partial_{i} \Psi_{j}^{1}=\beta_{i j} \Psi_{i}^{1}, \quad \partial_{j} \Psi_{j}^{0}=\Psi_{j}^{1}-\sum_{m \neq j} \beta_{m j} \Psi_{m}^{0} .
$$

Подчеркнем, что условия совместности этой расширенной системы уравнений полностью эквивалентны уравнениям (1.3) и (1.4).

В четвертом параграфе мы выделяем алгебро-геометрические данные, соответствующие егоровским метрикам, и получаем точную тэта-функциональную формулу для потенциалов $\Phi(u)$ построенных метрик.

Как уже отмечалось выше, в работе [5] была установлена связь задачи классификации егоровских метрик с задачей классификации моделей топологической теории поля. Последняя задача для модели, содержащей $n$ примарных полей $\phi_{1}, \ldots, \phi_{n}$, может быть сформулирована в терминах так называемых уравнений ассоциативности для статсуммы деформированной теории $[9,10]$. Соответствуюшие уравнения эквивалентны условиям того, что коммутативная алгебра 
с генераторами $\phi_{k}$ и структурными константами $c_{k l}^{m}(x)$, определяемыми третьими производными функции $F$ :

$$
\begin{gathered}
c_{k l m}(x)=\frac{\partial^{3} F(x)}{\partial x^{k} \partial x^{l} \partial x^{m}}, \\
\phi_{k} \phi_{l}=c_{k l}^{m}(x) \phi_{m}, \quad c_{k l}^{m}=c_{k l i} \eta^{i m}, \quad \eta_{k i} \eta^{i m}=\delta_{k}^{m},
\end{gathered}
$$

является ассоциативной алгеброй, т. е.

$$
c_{i j}^{k}(x) c_{k m}^{l}(x)=c_{j m}^{k}(x) c_{i k}^{l}(x) .
$$

Дополнительно требуется, чтобы существовали константы $r^{m}$, такие, что элементы постоянной матрицы $\eta$ в (1.13) равны

$$
\eta_{k l}=r^{m} c_{k l m}(x) .
$$

Равенства (1.14) являются переопределенной системой нелинейных уравнений на неизвестную функцию $F$. Оказывается, что для любого решения системы $(1.14)$, (1.15), такого, что соответствующая алгебра (1.13) полупроста, существует егоровская метрика, такая, что третьи производные статсуммы равны

$$
c_{k l m}=\sum_{i=1}^{n} H_{i}^{2} \frac{\partial u^{i}}{\partial x^{k}} \frac{\partial u^{i}}{\partial x^{l}} \frac{\partial u^{i}}{\partial x^{m}} .
$$

Верно и обратное утверждение. А именно, для любого набора симметрических коэффищиентов вращения $\beta_{i j}=\beta_{j i}$, удовлетворяющих (1.3) и (1.4), существует $n$-параметрическое семейство егоровских метрик, таких, что функции, определяемые равенствами (1.16), являются третьими производными некоторой функции $F$. (Напомним, что каждому набору коэффициентов вращения отвечает бесконечно много плоских диагональных метрик.)

В последнем параграфе мы явно определяем для любой алгебро-геометрической егоровской метрики функцию $F$, такую, что ее третьи производные имеют вид (1.16) и удовлетворяют уравнениям (1.14). Эти уравнения являются усеченной системой уравнений ассоциативности. На следующем шаге мы выделяем те метрики, для которых выполнено и соотношение (1.15).

\section{§2. Билинейные соотношения для функций Бейкера-Ахиезера и плоские диагональные метрики}

В начале этого параграфа мы приведем необходимые сведения из теории алгебро-геометрического интегрирования нелинейных уравнений. Общая схема алгебро-геометрического интегрирования была предложена автором [11, 12]. В ее основе лежит понятие функций Бейкера-Ахиезера, которые определяются своими аналитическими свойствами на вспомогательных римановых поверхностях.

Рассмотрим гладкую алгебраическую кривую $\Gamma$ рода $g$ с фиксированными локальными координатами $w_{i}(Q)$ в окрестностях $n$ отмеченных точек $P_{i}, i=$ $1, \ldots, n$, на $\Gamma, w_{i}\left(P_{i}\right)=0$. Для любого набора $R$ из $l$ точек $R_{\alpha}, \alpha=1, \ldots, l$, 
и для любого набора $D$ из $g+l-1$ точек $\gamma_{1}, \ldots, \gamma_{g+l-1}$ в общем положении существует единственная функция $\psi(u, Q \mid D, R), u=\left(u_{1}, \ldots, u_{n}\right), Q \in \Gamma$, такая, что:

$\left(1^{0}\right) \psi(u, Q \mid D, R)$ как функция переменной $Q \in \Gamma$ является мероморфной вне отмеченных точек $P_{j}$ и имеет не более чем простые полюсы в точках $\gamma_{s}$ (если все они различны);

$\left(2^{0}\right)$ в окрестности точки $P_{j}$ функция $\psi$ имеет вид

$$
\psi=e^{u^{j} w_{j}^{-1}}\left(\sum_{s=0}^{\infty} \xi_{s}^{j}(u) w_{j}^{s}\right), \quad w_{j}=w_{j}(Q) ;
$$

$\left(3^{0}\right) \psi$ удовлетворяет условиям нормировки

$$
\psi\left(u, R_{\alpha}\right)=1 .
$$

В дальнейшем мы будем часто обозначать функцию Бейкера-Ахиезера через $\psi(u, Q)$, опуская для краткости явное указание на ее зависимость от дивизоров $D=\gamma_{1}+\cdots+\gamma_{g+l-1}$ и $R=R_{1}+\cdots+R_{l}$.

Явные выражения функций Бейкера-Ахиезера через тэта-функции Римана были получены в [12] как обобщения формул, предложенных в работе [13] для блоховских решений конечнозонных операторов Шрёдингера.

Тэта-функцией Римана, соответствующей алгебраической кривой $\Gamma$ рода $g$, называется целая функция $g$ комплексных переменных $z=\left(z_{1}, \ldots, z_{g}\right)$, задаваемая своим рядом Фурье

$$
\theta\left(z_{1}, \ldots, z_{g}\right)=\sum_{m \in \mathbb{Z}^{g}} e^{2 \pi i(m, z)+\pi i(B m, m)},
$$

где $B=\left(B_{i j}\right)$ - матрица $b$-периодов нормированных голоморфных дифференциалов $\omega_{j}(P)$ на $\Gamma$ :

$$
B_{i j}=\oint_{b_{i}} \omega_{j}, \quad \oint_{a_{j}} \omega_{i}=\delta_{i j} .
$$

Здесь $a_{i}, b_{i}$ - базис циклов на $\Gamma$ с канонической матрицей пересечений $a_{i} \cdot a_{j}=$ $b_{i} \cdot b_{j}=0, a_{i} \cdot b_{j}=\delta_{i j}$.

Вектор $A(P)$ с координатами $A_{k}(Q)=\int_{q_{0}}^{Q} \omega_{k}$ определяет так называемое отображение Абеля.

Как следует из теоремы Римана-Роха, для дивизоров $D$ и $R$ в общем положении существует и единственна мероморфная функция $r_{\alpha}(Q)$, такая, что ее дивизор полюсов совпадает с дивизором $D$ и $r_{\alpha}\left(R_{\beta}\right)=\delta_{\alpha, \beta}$. Эта функция может быть представлена в следующем виде (см. подробнее в [14]):

$$
r_{\alpha}(Q)=\frac{f_{\alpha}(Q)}{f_{\alpha}\left(R_{\alpha}\right)}, \quad f_{\alpha}(Q)=\theta\left(A(Q)+Z_{\alpha}\right) \frac{\prod_{\beta \neq \alpha} \theta\left(A(Q)+F_{\beta}\right)}{\prod_{m=1}^{l} \theta\left(A(Q)+S_{m}\right)},
$$

Где

$$
\begin{gathered}
F_{\beta}=-\mathscr{K}-A\left(R_{\beta}\right)-\sum_{s=1}^{g-1} A\left(\gamma_{s}\right), \quad S_{m}=-\mathscr{K}-A\left(\gamma_{g-1+m}\right)-\sum_{s=1}^{g-1} A\left(\gamma_{s}\right), \\
Z_{\alpha}=Z_{0}-A\left(R_{\alpha}\right), \quad Z_{0}=-\mathscr{K}-\sum_{s=1}^{g+l-1} A\left(\gamma_{s}\right)+\sum_{\alpha=1}^{l} A\left(R_{\alpha}\right)
\end{gathered}
$$

и $\mathscr{K}$ - вектор римановых констант. 
Рассмотрим единственный нормированный мероморфный дифференциал $d \Omega_{j}$ на $Г$, голоморфный вне точки $P_{j}$ и имеющий в окрестности этой точки вид $d \Omega_{j}=d\left(w_{j}^{-1}+O\left(w_{j}\right)\right)$. Он задает вектор $V^{(j)}$ с координатами $V_{k}^{(j)}=\frac{1}{2 \pi i} \oint_{b_{k}} d \Omega_{j}$.

ТЕорема 2.1. Функиия Бейкера-Ахиезера $\psi(u, Q \mid D, R)$ имеет вид

$$
\psi=\sum_{\alpha=1}^{l} r_{\alpha}(Q) \frac{\theta\left(A(Q)+\sum_{i=1}^{n}\left(u^{i} V^{(i)}\right)+Z_{\alpha}\right) \theta\left(Z_{0}\right)}{\theta\left(A(Q)+Z_{\alpha}\right) \theta\left(\sum_{i=1}^{n}\left(u^{i} V^{(i)}\right)+Z_{0}\right)} \exp \left(\sum_{i=1}^{n} u^{i} \int_{R_{\alpha}}^{Q} d \Omega_{i}\right) .
$$

Допустимые кривые. Как будет показано ниже, алгебро-геометрические плоские диагональные метрики строятся с помощью функций Бейкера-Ахиезера, отвечающих специальным алгебро-геометрические данным, которые в дальнейшем мы будем называть допустимыми.

Допустимая алгебраическая кривая $\Gamma$ должна обладать голоморфной инволюцией $\sigma: \Gamma \rightarrow \Gamma$, имеющей $2 m \geqslant n$ неподвижных точек $P_{1}, \ldots, P_{n}, Q_{1}, \ldots$, $Q_{2 m-n}, m \leqslant n$. Локальные координаты $w_{j}(Q)$ в окрестностях точек $P_{1}, \ldots, P_{n}$ должны быть нечетными относительно инволюции:

$$
w_{j}(Q)=-w_{j}(\sigma(Q))
$$

Факторкривая $\Gamma_{0}=\Gamma / \sigma$ является гладкой алгебраической кривой. Проекция $\pi: \Gamma \rightarrow \Gamma_{0}=\Gamma / \sigma$ представляет $\Gamma$ как двулистное накрытие над $\Gamma_{0}$ с $2 m$ точками ветвления $P_{j}, Q_{s}$. В этой реализации инволюция $\sigma$ является просто перестановкой листов. Для $Q \in \Gamma$ точка $\sigma(Q)$ будет обозначаться через $Q^{\sigma}$.

Из теоремы Римана-Гурвица следует соотношение $g=2 g_{0}-1+m$, где $g_{0}$ - род кривой $\Gamma_{0}$.

Допустимые дивизоры. Зафиксируем на $\Gamma_{0}$ дополнительно набор из $n-m$ точек $\widehat{Q}_{1}, \ldots, \widehat{Q}_{n-m}$. Пара дивизоров $D$ и $R$ на $\Gamma$ будет называться допустимой, если на $\Gamma_{0}$ существует мероморфный дифференциал $d \Omega_{0}$, такой, что

(a) $d \Omega_{0}(P), P \in \Gamma_{0}$, имеет $m+l$ простых полюсов в точках $Q_{1}, \ldots, Q_{2 m-n}$, $\widehat{Q}_{1}, \ldots, \widehat{Q}_{n-m}$ и точках $\widehat{R}_{\alpha}=\pi\left(R_{\alpha}\right)$;

(b) дифференциал $d \Omega_{0}$ обращается в нуль в проекциях $\widehat{\gamma}_{s}$ точек дивизора $D$,

$$
d \Omega_{0}\left(\widehat{\gamma}_{s}\right)=0, \quad \widehat{\gamma}_{s}=\pi\left(\gamma_{s}\right) .
$$

Дифференциал $d \Omega_{0}$ можно рассматривать как четный мероморфный дифференциал на накрывающей $\Gamma$, где он имеет $n+2 l$ простых полюсов в точках ветвления $Q_{1}, \ldots, Q_{2 m-n}$ и в прообразах остальных полюсов этого дифференциала на $\Gamma_{0}$. Обозначим прообразы точек $\widehat{Q}_{k}$ через $Q_{2 m-n+1}, \ldots, Q_{2 m}$ :

$$
\pi\left(Q_{2 m-n+i}\right)=\pi\left(Q_{n-i+1}\right)=\widehat{Q}_{i}, \quad i=1, \ldots, n-m .
$$

Инволюция $\sigma$ индуцирует инволюцию $\sigma(k)$ индексов, нумерующих точки $Q_{k}$, $\sigma\left(Q_{k}\right)=Q_{\sigma(k)}$ : $\sigma(k)=k, \quad k=1, \ldots, 2 m-n, \quad \sigma(k)=2 m-k+1, \quad k=2 m-n+1, \ldots, n$. 
Из определения допустимых пар дивизоров следует, что их классы линейной эквивалентности удовлетворяют соотношению

$$
D+D^{\sigma}-R-R^{\sigma}=K+\sum_{j=1}^{n}\left(Q_{j}-P_{j}\right) .
$$

ПримеР. Гиперэллиптические кривые. Простейшими примерами допустимых кривых являются гиперэллиптические кривые. Рассмотрим гиперэллиптическую кривую $Г$, заданную уравнением

$$
\lambda^{2}=\frac{\prod_{j=1}^{2 m-n}\left(E-Q_{j}\right) \prod_{k=1}^{n-m}\left(E-\widehat{Q}_{k}\right)^{2}}{\prod_{i=1}^{n}\left(E-P_{i}\right)}, \quad m \leqslant n .
$$

Здесь $P_{i}, Q_{j}, \widehat{Q}_{k}$ - комплексные числа. Род кривой $\Gamma$ равен $g=m-1$. Любой набор из $m+l-2$ точек $\gamma_{s}, \gamma_{s} \neq \gamma_{s^{\prime}}$, и любой набор из $l$ точек являются допустимой парой дивизоров. Соответствующий дифференциал равен

$$
d \Omega_{0}=\frac{\prod_{s=1}^{m+l-2}\left(E-\gamma_{s}\right)}{\prod_{j=1}^{2 m-n}\left(E-Q_{j}\right) \prod_{k=1}^{n-m}\left(E-\widehat{Q}_{k}\right) \prod_{\alpha=1}^{l}\left(E-R_{\alpha}\right)} d E .
$$

Как будет показано в дальнейшем, плоские диагональные метрики, соответствующие гиперэллиптическим кривым, являются егоровскими метриками. Более того, в заключительном параграфе будет показано, что гиперэллиптическим кривым отвечают и простейшие решения уравнений ассоциативности.

ВАЖНОЕ ЗАМЕЧАНИЕ. В дальнейшей основной части работы мы для простоты формул будем предполагать, если не оговорено противное, что дивизор $R$ и дивизор точек $Q_{j}$ находятся в общем положении и не пересекаются друг с другом. В последнем параграфе мы специально рассмотрим случай, когда эти дивизоры совпадают.

Tеорема 2.2. Рассмотрим функиию Бейкера-Ахиезера $\psi(u, Q \mid D, R)$, отвечающую допустимой кривой и допустимой паре дивизоров $D u R$. Тогда бункиии $x^{j}(u)=\psi\left(u, Q_{j}\right), j=1, \ldots, n$, удовлетворяют соотношению

$$
\sum_{k, l} \eta_{k l} \partial_{i} x^{k} \partial_{j} x^{l}=\varepsilon_{i}^{2} h_{i}^{2} \delta_{i j}
$$

где $h_{i}=\xi_{0}^{i}(u)$ равны первым коэффичиентам разложений (2.1), константы $\varepsilon_{i}^{2}$ определяются разложением дифференциала $d \Omega_{0}$ в точках $P_{i}$ :

$$
d \Omega_{0}=\frac{1}{2}\left(\varepsilon_{i}^{2}+O\left(w_{i}^{0}\right)\right) d w_{i}^{0}=w_{i}\left(\varepsilon_{i}^{2}+O\left(w_{i}^{2}\right)\right) d w_{i},
$$

u, наконец,

$$
\eta_{k l}=\eta_{k} \delta_{k, \sigma(l)}, \quad \eta_{k}=\underset{Q_{k}}{\operatorname{res}} d \Omega_{0} .
$$

ДокАЗАТЕЛЬСтво. Рассмотрим дифференциал

$$
d \Omega_{i j}^{(1)}(u, Q)=\partial_{i} \psi(u, Q) \partial_{j} \psi(u, \sigma(Q)) d \Omega_{0}(\pi(Q)) .
$$


Из определения допустимых данных следует, что для $i \neq j$ он является мероморфным дифференциалом с полюсами в точках $Q_{1}, \ldots, Q_{n}$. Действительно, полюсы первых двух сомножителей $\partial_{i} \psi_{i}(u, Q)$ и $\partial_{j} \psi(u, \sigma(Q))$ в точках $\gamma_{s}$ и $\sigma\left(\gamma_{s}\right)$ сокращаются с нулями дифференциала $d \Omega_{0}$. Существенные особенности этих же сомножителей в точках $P_{k}$ сокращаются друг с другом. Кроме того, простые полюсы произведения первых двух сомножителей в точках $P_{i}$ и $P_{j}$ сокращаются с нулями $d \Omega_{0}$, рассматриваемого как дифференциал на $Г$; см. (2.6). Наконец, $d \Omega^{(1)}$ не имеет полюсов в точках $R_{\alpha}$ и $R_{\alpha}^{\sigma}$ в силу нормировочных условий (2.2). Сумма всех вычетов мероморфного дифференциала на компактной римановой поверхности должна равняться нулю. Следовательно,

$$
\sum_{k=1}^{n} \underset{Q_{k}}{\operatorname{res}} d \Omega_{i j}^{(1)}=0, \quad i \neq j
$$

Левая часть этого равенства совпадает с левой частью равенства (2.5).

В случае $i=j$ дифференщиал $d \Omega_{i i}^{(1)}$ имеет дополнительно простой полюс в точке $P_{i}$ с вычетом $\operatorname{res}_{P_{i}} d \Omega_{i i}^{(1)}=-\varepsilon_{i}^{2} h_{i}^{2}$. Последнее равенство доказывает $(2.5)$ для $i=j$ и завершает доказательство теоремы.

СлЕДСТВИЕ 2.1. Для любого набора допустимых данных $\left\{\Gamma, P_{i}, Q_{j}, D, R\right\}$ формула

$$
H_{i}(u)=\varepsilon_{i} \sum_{\alpha=1}^{l} r_{\alpha}\left(P_{i}\right) \frac{\theta\left(A\left(P_{i}\right)+\sum_{i=1}^{n}\left(u^{i} V^{(i)}\right)+Z_{\alpha}\right) \theta\left(Z_{0}\right)}{\theta\left(A\left(P_{i}\right)+Z_{\alpha}\right) \theta\left(\sum_{i=1}^{n}\left(u^{i} V^{(i)}\right)+Z_{0}\right)} \exp \left(\sum_{j=1}^{n} \omega_{i j}^{\alpha} u^{j}\right),
$$

где функиия $r_{\alpha}(Q)$ определена равенством (2.3),

$$
\omega_{i j}^{\alpha}=\int_{R_{\alpha}}^{P_{i}} d \Omega_{j}, \quad i \neq j, \quad \omega_{i i}^{\alpha}=\lim _{Q \rightarrow P_{i}}\left(\int_{R_{\alpha}}^{Q} d \Omega_{i}-w_{i}^{-1}(Q)\right),
$$

определяет коэффициенть плоской диагональной метрики. Соответствующие плоские координать даются формулами

$$
\begin{gathered}
x^{k}(u)=\sum_{\alpha=1}^{l} r_{\alpha}\left(Q_{k}\right) x_{\alpha}^{k}(u) \\
x_{\alpha}^{k}(u)=\frac{\theta\left(A\left(Q_{k}\right)+\sum_{i=1}^{n}\left(u^{i} V^{(i)}\right)+Z_{\alpha}\right) \theta\left(Z_{0}\right)}{\theta\left(A\left(Q_{k}\right)+Z_{\alpha}\right) \theta\left(\sum_{i=1}^{n}\left(u^{i} V^{(i)}\right)+Z_{0}\right)} \exp \left(\sum_{i=1}^{n} u^{i} \int_{R_{\alpha}}^{Q_{k}} d \Omega_{i}\right) .
\end{gathered}
$$

Условия вещественности. В общем случае коэффициенты $H_{i}(u)$ построенных алгебро-геометрических плоских диагональных метрик и соответствующие им плоские координаты являются мероморфными функциями переменных $u^{i}$. Приведем условия на алгебро-геометрические данные, достаточные для того, чтобы соответствующие коэффициенты метрик были вещественными функциями вещественныл переменных $u^{i}$.

Пусть $\Gamma_{0}$ - вещественная алгебраическая кривая, т. е. кривая с антиголоморфной инволюцией $\tau_{0}: \Gamma_{0} \rightarrow \Gamma_{0}$, и пусть точки $\left\{P_{1}, \ldots, P_{n}\right\}$ и $\left\{Q_{1}, \ldots\right.$, $\left.Q_{2 m-n}\right\}$ являются неподвижными точками инволющии $\tau_{0}$. В этом случае $\tau_{0}$ индуцирует антиголоморфную инволюцию $\tau$ кривой Г. Мы предполагаем, что 
локальные координаты $w_{j}$ в точках $P_{j}$ удовлетворяют условию $w_{j}(\tau(Q))=$ $\overline{w_{j}(Q)}$. Предположим также, что наборы точек $Q_{k}$ и дивизоры $D, R$ инвариантны относительно $\tau$ :

$$
\tau\left(Q_{j}\right)=Q_{\kappa(j)}, \quad \tau\left(R_{\alpha}\right)=R_{\kappa_{1}(\alpha)}, \quad \tau\left(\gamma_{s}\right)=\gamma_{\kappa_{2}(s)} .
$$

Здесь $\kappa_{i}(\cdot)$ - соответствующие перестановки индексов.

ТЕОРемА 2.3. Предположим, что набор алгебро-геометрических данных является вещественным. Тогда соответствуюшая функиия Бейкера-Ахиезера удовлетворяет равенству

$$
\psi(u, Q \mid D, R)=\overline{\psi(u, \tau(Q) \mid D, R)}
$$

и формула (2.8) определяет вещественную плоскую диагональную метрику.

Сигнатура соответствующей метрики зависит от инволюций $\kappa(j)$ и $\kappa_{1}(\alpha)$. Варьируя выбор начальных данных, можно получить плоские диагональные метрики в любых псевдоевклидовых пространствах $R^{p, q}$. В общем случае метрики являются сингулярными при некоторых значениях переменных $u^{i}$. Для получения гладких метрик следует еше ограничить начальные данные. Техника получения подобных ограничений относительно стандартна в теории конечнозонного интегрирования. Мы планируем рассмотреть этот вопрос подобнее в дальнейшем.

\section{§3. Дифференциальные уравнения для функции Бейкера-Ахиезера}

В этом параграфе мы поясним значение наших ограничений на алгебро-геометрические данные с точки зрения дифференциальных уравнений для функции Бейкера-Ахиезера.

Следующее утверждение — простое обобщение результатов работы [17], в которой для случая $n=2$ было доказано, что соответствующие функции БейкераАхиезера являются решениями двумерного оператора Шрёдингера.

Лемма 3.1. Функиия Бейкера-Ахиезера $\psi(u, Q \mid D, R)$ удовлетворяет уравнениям

$$
\partial_{i} \partial_{j} \psi=c_{i j}^{i} \partial_{i} \psi+c_{i j}^{j} \partial_{j} \psi, \quad i \neq j
$$

$2 \partial e$

$$
c_{i j}^{i}(u)=\partial_{j} h_{i} / h_{i}, \quad c_{i j}^{j}(u)=\partial_{i} h_{j} / h_{j}
$$

$u h_{i}(u)=\xi_{0}^{i}(u)$ являются первыли коэффиииентами разложения (2.1).

Уравнения (3.1) имеют вид уравнений (1.7), представляющих собой часть уравнений для плоских координат метрики с коэффициентами $H_{i}(u)=\varepsilon_{i} h_{i}(u)$, где $\varepsilon_{i}$ - константы. Приведем теперь дополнительные уравнения, которым удовлетворяют функции Бейкера-Ахиезера и которые в случае допустимых алгеброгеометрических данных превращаются в уравнения (1.8).

Рассмотрим алгебро-геометрические данные $\left\{\Gamma, P_{j}, w_{j}, \gamma_{s}, R_{\alpha}\right\}$, задающие функцию Бейкера-Ахиезера $\psi(u, Q \mid D, R)$. Зафиксируем дополнительно набор 
из $n$ точек $Q_{1}, \ldots, Q_{n}$. Тогда в случае общего положения существует единственная функция $\psi^{1}=\psi^{1}(u, Q \mid D, R)$, такая, что

$\left(1^{1}\right) \psi^{1}(u, Q)$ как функция переменной $Q \in \Gamma$ мероморфна вне отмеченных точек $P_{j}$, имеет не более чем простые полюсы в точках $\gamma_{s}$ и равна нулю в точках $Q_{1}, \ldots, Q_{n}$ :

$$
\psi^{1}\left(u, Q_{k}\right)=0
$$

$\left(2^{1}\right)$ в окрестности точки $P_{j}$ функция $\psi^{1}$ имеет вид

$$
\psi^{1}=w_{j}^{-1} e^{u^{j} w_{j}^{-1}}\left(\sum_{s=0}^{\infty} \xi_{1, s}^{j}(u) w_{j}^{s}\right), \quad w_{j}=w_{j}(Q)
$$

$\left(3^{1}\right) \psi^{1}$ удовлетворяет условиям нормировки $\psi^{1}\left(u, R_{\alpha} \mid D, R\right)=1$.

ЛЕммА 3.2. Функиии $\psi(u, Q \mid D, R) \quad u \quad \psi^{1}(u, Q \mid D, R)$ удовлетворяют уравнениям

$2 \partial e$

$$
\partial_{i}^{2} \psi-c_{i}^{1} \partial_{i} \psi^{1}+\sum_{j=1}^{n} v_{i j} \partial_{j} \psi=0
$$

$$
\begin{gathered}
c_{i}^{1}=\frac{h_{i}}{h_{i}^{1}}, \quad v_{i i}=\frac{\partial_{i} h_{i}^{1}}{h_{i}^{1}}-2 \frac{\partial_{i} h_{i}}{h_{i}}+\frac{g_{i}^{1}}{h_{i}^{1}}-\frac{g_{i}}{h_{i}}, \\
v_{i j}=\frac{h_{i}}{h_{j}} \frac{\partial_{i} h_{j}^{1}}{h_{i}^{1}}, \quad i \neq j,
\end{gathered}
$$

и функиии $h_{i}=\xi_{0}^{i}, h_{i}^{1}=\xi_{1,0}^{i}, g_{i}=\xi_{1}^{i}, g_{i}^{1}=\xi_{1,1}^{i}$ равньи первылм коэффициентам разложений (2.1) $и$ (3.2).

Для доказательства рассмотрим функцию, определенную левой частью равенства (3.3). Из формул (3.4) и (3.5) следует, что она удовлетворяет первым двум условиям, определяющим функцию $\psi$, и равна нулю во всех точках $R_{\alpha}$. Следовательно, она тождественно равна нулю.

Рассмотрим теперь случай допустимых алгебро-геометрических данных. (В этом случае набор точек $Q_{i}$ в определении функции $\psi^{1}$ является тем же набором, который фигурирует в определении допустимых пар дивизоров, т.е. $Q_{1}, \ldots, Q_{2 m-n}$ - точки ветвления, а $Q_{2 m-n+1}, \ldots, Q_{2 n}$ - прообразы точек $\widehat{Q}_{k} \cdot$ )

Теорема 3.1. Функиии Бейкера-Ахиезера $\psi(u, Q \mid D, R) u \psi^{1}(u, Q \mid D, R)$, отвечающие допустимьм алаебро-геометрическим данньм, удовлетворяют уравнениям (3.1) и уравнениям

$$
\partial_{i}^{2} \psi=c_{i}^{1} \partial_{i} \psi^{1}+\sum_{j=1}^{n} \Gamma_{i i}^{j} \partial_{j} \psi
$$

Здесь $\Gamma_{i i}^{j}$ - символь Кристоффеля (1.9) метрики $H_{i}(u)=\varepsilon_{i} h_{i}(u)$.

ДокАЗАТЕльСТво. Дифференциал

$$
d \Omega_{i j}^{(2)}=\partial_{i} \psi^{1}(u, Q) \partial_{j} \psi\left(u, Q^{\sigma}\right) d \Omega_{0}(\pi(Q))
$$


голоморфен вне точек $P_{i}$ и $P_{j}$. Его вычеты в этих точках равны

$$
\underset{P_{i}}{\operatorname{res}} d \Omega_{i j}^{(2)}=\varepsilon_{i}^{2} h_{i}^{1} \partial_{j} h_{i}, \quad \underset{P_{j}}{\operatorname{res}} d \Omega_{i j}^{(2)}=-\varepsilon_{j}^{2} h_{j} \partial_{i} h_{j}^{1} .
$$

Следовательно, $\varepsilon_{i}^{2} h_{i}^{1} \partial_{j} h_{i}=\varepsilon_{j}^{2} h_{j} \partial_{i} h_{j}^{1}$. Из последней формулы вытекает, что коэффициенты $v_{i j}$ в $(3.5)$ для $i \neq j$ равны $\Gamma_{i i}^{j}$.

Дифференциал $d \Omega_{i i}^{1}$ имеет единственный полюс в точке $P_{i}$. Следовательно, его вычет в этой точке равен нулю:

$$
\underset{P_{i}}{\operatorname{res}} d \Omega_{i i}^{1}=h_{i}^{1}\left(g_{i}+\partial_{i} h_{i}\right)-h_{i}\left(g_{i}^{1}+\partial_{i} h_{i}^{1}\right)=0 .
$$

Из (3.7) следует, что коэффициент $v_{i i}$, заданный формулой $(3.4)$, равен $\Gamma_{i i}^{i}$.

Отметим еще раз, что в точках $Q_{j}$ равенство (3.6) совпадает с (1.8).

СЛЕДСТВИЕ 3.1. Функиии

$$
\Psi_{i}^{0}(u, Q)=\frac{1}{h_{i}(u)} \partial_{i} \psi(u, Q), \quad \Psi_{i}^{1}(u, Q)=\frac{1}{h_{i}^{1}(u)} \partial_{i} \psi^{1}(u, Q)
$$

удовлетворяют уравнениям (1.11), где $\beta_{i j}(u)$ являются коэффициентами врашения (1.5) метрики $H_{i}(u)$.

Доказательство следствия вытекает из простой подстановки (3.8) в (3.1) и (3.6).

Из определения функции Бейкера-Ахиезера следует, что функции $\Psi_{i}^{0}(u, Q)$ и $\Psi_{i}^{1}(u, Q)$ имеют следующие аналитические свойства на кривой $\Gamma$ :

$\left(1^{2}\right) \Psi_{i}^{N}(u, Q), N=0,1$ мероморфны вне точек $P_{j}$ и имеют не более чем простые полюсы в точках $\gamma_{1}, \ldots, \gamma_{g+l-1}$;

$\left(2^{2}\right)$ в окрестности точки $P_{j}$ функция $\Psi_{i}^{N}$ имеет вид

$$
\Psi_{i}^{N}=w_{j}^{-N-1} e^{u_{j} w_{j}^{-1}}\left(\delta_{i j}+\sum_{s=1}^{\infty} \zeta_{s, N}^{i j}(u) w_{j}^{s}\right), \quad w_{j}=w_{j}(Q)
$$

$\left(3^{2}\right)$ функции $\Psi_{i}^{N}$ равны нулю в точках $R_{\alpha}$, а функции $\Psi_{i}^{1}$, кроме того, равны нулю в точках $Q_{j}$ :

$$
\Psi_{i}^{N}\left(u, R_{\alpha}\right)=0, \quad \Psi_{i}^{1}\left(u, Q_{j}\right)=0
$$

Лемма 3.3. Пусть Г является гладкой алгебраической кривой рода $g$ c Фиксированными $2 n$ точками $P_{j}, Q_{j}$ и биксированньли локальньми координатами $w_{j}(Q)$ в окрестностях точек $P_{j}$. Тогда для любого набора $g+l-1$ точек $\gamma_{s}$ в общем положении существуют единственнье функиии $\Psi_{i}^{0}(u, Q)$ $u \Psi_{i}^{1}(u, Q)$, удовлетворяюшие сформулированным выше условиям $\left(1^{2}\right)-\left(3^{2}\right)$.

При заданной допустимой кривой $\Gamma$ с отмеченными точками $P_{i}, Q_{i}$ и фиксированными локальными координатами $w_{i}$ функции Бейкера-Ахиезера и соответствующие коэффициенты $H_{i}(u \mid D, R)$ плоской диагональной метрики зависят от допустимой пары дивизоров $D$ и $R$. Две пары дивизоров $D, R$ и $D^{\prime}, R^{\prime}$ будем называть эквивалентными, если линейно эквивалентны их разности $D-R$ 
и $D^{\prime}-R^{\prime}$, т. е. если существует мероморфная функция $f(Q)$ на $\Gamma$, такая, что дивизор ее полюсов и дивизор ее нулей равны

$$
(f)_{\infty}=D+R^{\prime}, \quad(f)_{0}=D^{\prime}+R
$$

соответственно. Из утверждения леммы 3.3 следует справедливость такого утверждения:

СлЕдСТВИЕ 3.2. Коэффиииенть вращения $\beta_{i j}(u \mid D, R) u \beta_{i j}\left(u \mid D^{\prime}, R^{\prime}\right)$, соответствующие эквивалентньм парам дивизоров, удовлетворяют соотношениям

$$
f\left(P_{i}\right) \beta_{i j}(u \mid D, R)=f\left(P_{j}\right) \beta_{i j}\left(u \mid D^{\prime}, R^{\prime}\right),
$$

где $f(Q)$ - функиия, такая, что выполнено (3.10).

Выразим коэффициенты вращения в терминах лишь функции $\Psi_{i}^{1}(u, Q \mid D, R)$.

ТЕОРемА 3.2. Коэффичиенть вращения $\beta_{i j}(u)$ алгебро-геометрической метрики с коэффичиентами $H_{i}(u \mid D, R)$ равнь

$$
\beta_{i j}(u \mid D, R)=\zeta_{1,1}^{j i}(u \mid D, R),
$$

где $\zeta_{1,1}^{j i}$ является первым коэффициентом разложения (3.9) функции $\Psi_{i}^{1}(u, Q \mid D, R)$. Коэффичиентьл Ламе $H_{i}\left(u \mid D, R, r^{\prime}\right)$ равнь

$2 \partial e$

$$
H_{i}(u \mid D, R)=-\sum_{\alpha} d_{\alpha} \Psi_{i}^{1}\left(u, R_{\alpha}^{\sigma} \mid D, R\right),
$$

$$
d_{\alpha}=\underset{R_{\alpha}}{\operatorname{res}} d \Omega_{0} .
$$

ДокАЗАТЕЛЬСтво. Из уравнений (1.11) следует, что $\Psi_{i}^{1} \quad$ удовлетворяют уравнению

$$
\partial_{i} \Psi_{j}^{1}=\beta_{i j} \Psi_{i}^{1}, \quad i \neq j
$$

Формула (3.11) непосредственно следует из (3.9) и (3.14). Для доказательства (3.12) рассмотрим дифференциал

$$
d \Omega_{i}^{(3)}(u, Q)=\Psi_{i}^{1}(u, Q) \psi\left(u, Q^{\sigma}\right) d \Omega_{0} .
$$

Этот мероморфный дифференциал имеет полюсы в точках $P_{i}$ и $R_{\alpha}^{\sigma}$ и

$$
\underset{P_{i}}{\operatorname{res}} d \Omega_{i}^{(3)}=H_{i}(u)
$$

Вычеты этого дифференщиала в точках $R_{\alpha}^{\sigma}$ равны соответствующим членам суммы в правой части равенства (3.12). Поскольку сумма всех вычетов равна нулю, то теорема доказана.

\section{§4. Егоровские метрики}

В этом параграфе мы опишем алгебро-геометрические данные, соответствующие егоровским метрикам, т. е. метрикам с симметричными коэффициентами вращения $\beta_{i j}=\beta_{j i}$. 
Пусть $E(P)$ является мероморфной функцией на гладкой алгебраической кривой $\Gamma_{0}$ рода $g_{0}$, имеющей $n$ простых полюсов в точках $P_{i}, 2 m-n$ простых нулей в точках $Q_{1}, \ldots, Q_{2 m-n}$ и $n-m$ двойных нулей в точках $\widehat{Q}_{1}, \ldots, \widehat{Q}_{n-m}$. Риманова поверхность $\Gamma$ функции $\lambda=\sqrt{E(P)}$ является допустимой кривой в смысле определений предыдущего параграфа. Функция $\lambda=\lambda(Q)$ является нечетной на кривой $\Gamma$, где она имеет простые полюсы в точках $P_{i}$ и простые нули в точках $Q_{j}, j=1, \ldots, n$. Она определяет локальные координаты $w_{j}(Q)=\lambda^{-1}(Q)$ в окрестностях точек $P_{i}$.

Теорема 4.1. Пусть $D, R$ - допустимая пара дивизоров на римановой поверхности Г функиии $\lambda(Q)$. Тогда

$$
\beta_{i j}(u \mid D, R)=\beta_{j i}(u \mid D, R) .
$$

Потенциал егоровской метрики $H_{i}(u \mid D, R)$ задаетсл формулой

$$
\Phi(u \mid D, R)=\sum_{\alpha=1}^{n} \lambda\left(R_{\alpha}\right) d_{\alpha} \psi\left(u, R_{\alpha}^{\sigma}\right),
$$

где $d_{\alpha}$ равны вычетам дифференциала $d \Omega_{0}$ в $R_{\alpha} ;$ см. (3.13).

ДокАЗАТЕЛЬСТво. Для получения (4.1) достаточно рассмотреть дифференциал $\lambda(Q) \Psi_{i}^{0}(u, Q) \Psi_{j}^{0}(u, \sigma(Q)) d \Omega_{0}$, имеющий полюсы лишь в точках $P_{i}$ и $P_{j}$. Его вычеты в этих точках равны $\beta_{j i}$ и $-\beta_{i j}$. Для доказательства (4.2) рассмотрим дифференциал

$$
d \Omega_{i}^{(4)}=\lambda(Q) \psi(u, Q) \partial_{i} \psi(u, \sigma(Q)) d \Omega_{0} .
$$

Он имеет полюсы в точках $P_{i}$ и $R_{\alpha}$ с вычетами

$$
\underset{P_{i}}{\operatorname{res}} d \Omega_{i}^{(4)}=-H_{i}^{2}, \quad \underset{R_{\alpha}}{\operatorname{res}} d \Omega_{i}^{(3)}=d_{\alpha} \lambda\left(R_{\alpha}\right) \partial_{i} \psi\left(u, R_{\alpha}^{\sigma}\right) .
$$

Сумма этих вычетов равна нулю, что доказывает (4.2).

\section{§5. Решения уравнений ассоциативности}

Доказанная в [5] эквивалентность проблемы классификации топологических массивных моделей квантовой теории поля и проблемы построения коэффициентов вращения егоровских метрик не дает явного способа построения решений уравнений ассоциативности. В настоящем параграфе мы приведем выражения для статсумм таких моделей, отвечающих построенным выше симметричным коэффициентам вращения.

ТеОрема 5.1. Пусть $\psi(u, Q \mid D, R)$ является функиией Бейкера-Ахиезера, соответствующей римановой поверхности $\Gamma$ функции $\lambda(Q)$ и допустимой паре дивизоров $D, R$. Тогда функчия $F(x)=F(u(x))$, определеннал формулой

$$
F(u)=\frac{1}{2}\left(\sum_{k, l=1}^{n} \eta_{k l} x^{k}(u) y^{l}(u)-\sum_{\alpha} \frac{d_{\alpha}}{\lambda\left(R_{\alpha}\right)} \psi\left(u, R_{\alpha}^{\sigma}\right)\right),
$$


где $\left(\eta_{k l}\right)$ - постоянная матрича, определенная в (2.7), константы $d_{\alpha}$ определеньь равенствами (3.13), а

$$
x^{k}(u)=\psi\left(u, Q_{k}\right), \quad y^{k}=d \psi\left(u, Q_{k}\right) / d \lambda,
$$

удовлетворяет уравнению

$$
\frac{\partial^{3} F(x)}{\partial x^{k} \partial x^{l} \partial x^{m}}=c_{k l m}=\sum_{i=1}^{n} H_{i}^{2} \frac{\partial u^{i}}{\partial x^{k}} \frac{\partial u^{i}}{\partial x^{l}} \frac{\partial u^{i}}{\partial x^{m}} .
$$

При этом функиии

$$
c_{k l}^{m}=\sum_{i=1}^{n} \frac{\partial u^{i}}{\partial x^{k}} \frac{\partial u^{i}}{\partial x^{l}} \frac{\partial x^{m}}{\partial u^{i}}
$$

удовлетворяют уравнениям ассочиативности (1.14).

ДокаЗАТЕЛЬСТво. В окрестности точки $P_{i}$ функции

$$
\phi_{k}=\frac{\partial \psi}{\partial x^{k}}, \quad \phi_{k l}=\frac{\partial^{2} \psi}{\partial x^{k} \partial x^{l}}
$$

имеют вид

$$
\phi_{k}=\frac{\partial u^{i}}{\partial x^{k}} \lambda e^{\lambda u^{i}}\left(h_{i}+O\left(\lambda^{-1}\right)\right), \quad \phi_{k l}=\frac{\partial u^{i}}{\partial x^{k}} \frac{\partial u^{i}}{\partial x^{k}} \lambda^{2} e^{\lambda u^{i}}\left(h_{i}+O\left(\lambda^{-1}\right)\right) .
$$

Следовательно,

$$
c_{k l m}=\sum_{i=1}^{n} \underset{P_{i}}{\operatorname{res}} d \Omega_{k ; l m}, \quad d \Omega_{k ; l m}=\phi_{k}(u, Q) \phi_{l m}\left(u, Q^{\sigma}\right) \frac{d \Omega_{0}}{\lambda(Q)} .
$$

Из определения $x^{k}$ вытекает, что $\phi_{k}\left(u, Q_{m}\right)=\delta_{k m}, \phi_{k l}\left(u, Q_{m}\right)=0$. Значит, дифференциал $d \Omega_{k ; l m}$ вне точек $P_{i}$ имеет единственный полюс в точке $Q_{k}$. Отсюда

$$
\begin{aligned}
c_{k l m}=-\underset{Q_{k}}{\operatorname{res}} d \Omega_{k ; l m} & =-\underset{Q_{k}}{\operatorname{res}} \phi_{l m}\left(u, \sigma\left(Q_{k}\right)\right) \frac{d \Omega_{0}}{\lambda(Q)} \\
& =-\frac{\partial^{2}}{\partial x^{l} \partial x^{m}}\left(\underset{Q_{k}}{\operatorname{res}} \psi\left(u, \sigma\left(Q_{k}\right)\right)\right) \frac{d \Omega_{0}}{\lambda(Q)} .
\end{aligned}
$$

В точке $Q_{k}$ имеем

$$
\psi(u, \sigma(Q))=x^{\sigma(k)}-y^{\sigma(k)} \lambda+O\left(\lambda^{2}\right) .
$$

Кроме того,

Значит,

$$
d \Omega_{0}=\frac{d \lambda}{\lambda}\left(\eta_{k}+\eta_{k}^{1} \lambda+O\left(\lambda^{2}\right)\right) .
$$

$$
\underset{Q_{k}}{\operatorname{res}} \psi\left(u, \sigma\left(Q_{k}\right)\right) \frac{d \Omega_{0}}{\lambda}=\eta_{k}^{1} x^{\sigma(k)}-\eta_{k} y^{\sigma(k)} .
$$

Из определения $F$ следует, что

$$
2 \frac{\partial}{\partial x^{k}} F=\eta_{k} y^{\sigma(k)}+\sum_{l}^{n} \eta_{l} x^{l} \frac{\partial y^{\sigma(l)}}{\partial x^{k}}-\frac{d_{\alpha}}{\lambda\left(R_{\alpha}\right)} \frac{\partial \psi\left(u, R_{\alpha}\right)}{\partial x^{k}} .
$$


Рассмотрим дифференциал

$$
d \Omega_{k}^{(5)}=\frac{\partial \psi(u, Q)}{\partial x^{k}} \psi\left(u, Q^{\sigma}\right) \frac{d \Omega_{0}}{\lambda(Q)} .
$$

Его вычеты в точках $Q_{l}$ и $R_{\alpha}^{\sigma}$ равны

$$
\begin{aligned}
& \underset{Q_{l}}{\operatorname{res}} d \Omega_{k}^{(5)}=\eta_{l} x^{\sigma(l)} \frac{\partial y^{l}}{\partial x^{k}}+\delta_{l, \sigma(k)}\left(\eta_{k}^{1} x^{\sigma(k)}-\eta_{k} y^{\sigma(k)}\right), \\
& \underset{R_{\alpha}^{\sigma}}{\operatorname{res}} d \Omega_{k}^{(5)}=-\frac{d_{\alpha}}{\lambda\left(R_{\alpha}\right)} \frac{\partial \psi\left(u, R_{\alpha}^{\sigma}\right)}{\partial x^{k}} .
\end{aligned}
$$

Отсюда следует, что

$$
\sum_{l=1}^{n} \eta_{l} x^{l} \frac{\partial y^{\sigma(l)}}{\partial x^{k}}-\sum_{\alpha} \frac{d_{\alpha}}{\lambda\left(R_{\alpha}\right)} \frac{\partial \psi\left(u, R_{\alpha}\right)}{\partial x^{k}}=\eta_{k} y^{\sigma(k)}-\eta_{k}^{1} x^{\sigma(k)} .
$$

Окончательно,

$$
\frac{\partial}{\partial x^{k}} F=\eta_{k} y^{\sigma(k)}-\frac{1}{2} \eta_{k}^{1} x^{\sigma(k)} .
$$

Это равенство совместно с (5.3), (5.4) доказывает (5.1).

Лемма 5.1. Функиия Бейкера-Ахиезера $\psi(u, Q \mid D, R)$, соответствуюшая римановой поверхности функции $\lambda(Q)$ и допустимой паре дивизоров $D, R$, удовлетворяет уравнениям

$$
\frac{\partial^{2}}{\partial x^{k} \partial x^{l}} \psi-\lambda \sum_{m=1}^{n} c_{k l}^{m} \frac{\partial}{\partial x^{m}} \psi_{m}=0
$$

ДокАЗАТЕльство. Функция $\widetilde{\psi}$, определенная левой частью равенства $(5.5)$, вне точек $P_{j}$ имеет полюсы лишь в точках дивизора $D$ и равна нулю в точках $Q_{j}$. Из определения $c_{k l}^{m}$ следует, что мероморфный сомножитель разложения функции $\widetilde{\psi}$ в точках $P_{i}$ имеет вид $O\left(\lambda^{-1}\right)$. Следовательно, из единственности функции Бейкера-Ахиезера вытекает равенство $\widetilde{\psi}=0$.

Уравнения (1.14) являются следствиями условий совместности системы (5.5). Теорема доказана.

ЗАмечАниЕ. Уравнения (5.5) могут быть записаны в векторной форме:

$$
\frac{\partial}{\partial x^{k}} \widetilde{\Psi}_{l}=\lambda \sum_{m=1}^{n} c_{k l}^{m} \widetilde{\Psi}_{m}, \quad \widetilde{\Psi}_{k}=\frac{\partial \psi}{\partial x^{k}} .
$$

Система (5.6) с симметричными коэффициентами $c_{k l}^{m}=c_{l k}^{m}$ как вспомогательная линейная система для уравнений (1.14) была введена в [5].

Теперь мы рассмотрим специальный случай нашей конструкции, когда дивизор $R$ совпадает с дивизором $\mathscr{Q}$ точек $Q_{j}$. Как было отмечено в замечании перед теоремой 2.2 , использованное ранее предположение, что $R$ не пересекается с $\mathscr{Q}$, было принято лишь для простоты формул. 
В случае $R=\mathscr{Q}$ допустимые дивизоры $D$ определяются следующим образом. Дивизор $D=\gamma_{1}+\cdots+\gamma_{g+n-1}$ называется допустимым, если существует мероморфный дифференциал $d \Omega_{0}$ на $\Gamma_{0}$ с полюсами порядка 2 в точках $Q_{1}, \ldots$, $Q_{2 m-n}$ и полюсами порядка 3 в точках $\widehat{Q}_{k}$, являющихся двукратными нулями функции $E(P)$, такой, что $d \Omega_{0}\left(\pi\left(\gamma_{s}\right)\right)=0$. Дифференциал $d \Omega_{0}$, рассматриваемый как четный дифференциал на $\Gamma$, в точках $Q_{k}, k=1 \ldots, n$ (в которых $\left.\lambda\left(Q_{k}\right)=0\right)$, имеет вид

$$
d \Omega_{0}=\frac{d \lambda}{\lambda^{3}(P)}\left(\eta_{k}+O(\lambda)\right)
$$

В рассматриваемом специальном случае плоские координаты даются не значениями функщии $\psi$ в точках $Q_{k}$ (которые теперь равны 1 ), а следующими коэффициентами разложения.

Теорема 5.2. Пусть $\psi(x, Q \mid D, \mathscr{Q})$ является функиией Бейкера-Ахиезеpa, соответствующей допустимому дивизору $D$ на римановой поверхности функции $\lambda(Q)$. Тогда функция $F(x)=\widetilde{F}(u(x))$, где

$$
\widetilde{F}(u)=\frac{1}{2} \sum_{k=1}^{n} \eta_{k} x^{k}(u) y^{\sigma(k)}(u),
$$

$\eta_{k}=\operatorname{res}_{Q_{k}} \lambda^{2} d \Omega_{0}, a x^{k}(u)$ and $y^{k}(u)$ определяются из разложения

$$
\psi=1+x^{k}(u) \lambda+y^{k}(u) \lambda^{2}+O\left(\lambda^{3}\right),
$$

является решением уравнений ассочиативности (1.12)-(1.15), т.е. удовлетворяет уравнениям (5.1); функции $c_{k l}^{m}$, определенные формулой (5.2), удовлетворяют (1.14) и дополнительному соотношению

$$
\sum_{m=1}^{n} c_{k l m}(u)=\eta_{k l} .
$$

Доказательство того, что $x^{k}$ являются плоскими координатами метрики с коэффициентами Ламе $H_{i}=\varepsilon_{i} h_{i}(u)$, где $h_{i}(u)$ равны главным членам разложений соответствующей функции Бейкера-Ахиезера в точках $P_{i}$, полностью аналогично общему случаю. Аналогичны и доказательства всех, кроме последнего, утверждений теоремы. Равенство (5.7) вытекает из следующего утверждения.

Лемма 5.2. Функиия Бейкера-Ахиезера $\psi$, соответствующая данным теоремы 4.3, удовлетворлет равенству

$$
\sum_{s=1}^{n} \frac{\partial}{\partial u^{s}} \psi=\lambda \psi .
$$

Левая и правая части равенства (5.8) регулярны вне точек $P_{k}$ и имеют одинаковые главные члены разложений в этих точках. Из единственности функции Бейкера-Ахиезера следует, что они равны между собой. Равенство (5.8) в точках $Q_{m}$ дает

$$
\sum_{s=1}^{n} \frac{\partial x^{m}}{\partial u^{s}}=1
$$


Значит,

$$
\sum_{m=1}^{n} c_{k l m}(u)=\sum_{i=1}^{n} H_{i}^{2} \frac{\partial u^{i}}{\partial x^{k}} \frac{\partial u^{i}}{\partial x^{l}}=\eta_{k l}
$$

Теорема доказана.

Точные тэта-функциональные формулы для $F$ получаются в результате подстановок соответствующих выражений для функции Бейкера-Ахиезера.

ПримеР. Эллиптические решения. Рассмотрим простейшие эллиптические криволинейные координаты и решения уравнений ассоциативности, соответствующие $n=l=3, m=2$ в примере из $\S 2$.

Рассмотрим эллиптическую кривую $Г$ с периодами $2 \omega, 2 \omega^{\prime}, \operatorname{Im} \omega^{\prime} / \omega>0$. В таком представлении мы отождествляем $P_{i}$ с полупериодами $\omega_{i}$,

$$
P_{1}=\omega_{1}=\omega, \quad P_{2}=\omega_{2}=\omega^{\prime}, \quad P_{3}=\omega_{3}=-\omega-\omega^{\prime} .
$$

Точкам $Q_{j}$ отвечают в фундаментальном параллелограмме $\Gamma$ точки с координатами $Q_{1}=0, Q_{2}=z_{0}, Q_{3}=-z_{0}$. Для $g=1$ любые дивизоры $D$ и $R$ являются допустимыми. Соответствующий дифференциал имеет вид

$$
d \Omega_{0}=\eta_{0} \frac{\sigma(z-\omega) \sigma\left(z-\omega^{\prime}\right) \sigma\left(z+\omega+\omega^{\prime}\right)}{\sigma(z) \sigma\left(z+z_{0}\right) \sigma\left(z-z_{0}\right)} \prod_{s=1}^{l} \frac{\sigma\left(z-\gamma_{s}\right) \sigma\left(z+\gamma_{s}\right)}{\sigma\left(z-R_{s}\right) \sigma\left(z+R_{s}\right)} d z,
$$

где $\sigma(z)=\sigma\left(z \mid \omega, \omega^{\prime}\right)$ - классическая $\sigma$-функция Вейерштрасса. Вычеты этого дифференциала

$$
\underset{z=0}{\operatorname{res}} d \Omega_{0}=\eta_{1}, \quad \underset{z= \pm z_{0}}{\operatorname{res}} d \Omega_{0}=\eta_{2}
$$

являются коэффициентами плоской метрики $d s^{2}=\eta_{1}\left(d x^{1}\right)^{2}+\eta_{2}\left(d x^{2}\right)\left(d x^{3}\right)$. Функщия Бейкера-Ахиезера имеет вид

$$
\psi(u, z)=\prod_{s=1}^{l} \frac{\sigma\left(z-R_{s}\right)}{\sigma\left(z-\gamma_{s}\right)}\left[\sum_{\alpha=1}^{l} r_{\alpha} \frac{\sigma\left(z+U-R_{\alpha}\right)}{\sigma\left(z-R_{\alpha}\right) \sigma(U)} \exp \left(\Omega(u, z)-\Omega\left(u, R_{\alpha}\right)\right)\right],
$$

где

$$
\begin{gathered}
U=u^{1}+u^{2}+u^{3}, \\
\Omega(u, z)=u^{1}(\zeta(z-\omega)+\eta)+u^{2}\left(\zeta\left(z-\omega^{\prime}\right)+\eta^{\prime}\right)+u^{3}\left(\zeta\left(z+\omega+\omega^{\prime}\right)-\eta-\eta^{\prime}\right), \\
\zeta(z)=\frac{\sigma^{\prime}(z)}{\sigma(z)}, \quad \eta=\zeta(\omega), \quad \eta^{\prime}=\zeta\left(\omega^{\prime}\right), \quad r_{\alpha}=\frac{\prod_{s=1}^{l} \sigma\left(R_{\alpha}-\gamma_{s}\right)}{\prod_{s \neq \alpha} \sigma\left(R_{\alpha}-R_{s}\right)} .
\end{gathered}
$$

В общем случае, когда $R_{\alpha} \neq Q_{j}$, плоские координаты даются значениями функции $\psi$ в точках $Q_{j}$, т.е.

$$
x^{1}=\psi(u, 0), \quad x^{2}=\psi\left(u, z_{0}\right), \quad x^{3}=\psi\left(u,-z_{0}\right) .
$$


Коэффициенты Ламе равны

$$
\begin{aligned}
& H_{1}(u)=\varepsilon_{1} \sum_{\alpha=1}^{n} r_{\alpha} \frac{\sigma\left(U-R_{\alpha}\right)}{\sigma\left(\omega-R_{\alpha}\right) \sigma(U)} e^{U \eta}, \\
& H_{2}(u)=\varepsilon_{2} \sum_{\alpha=1}^{n} r_{\alpha} \frac{\sigma\left(\omega^{\prime}+U-R_{\alpha}\right)}{\sigma\left(\omega^{\prime}-R_{\alpha}\right) \sigma(U)} e^{U \eta^{\prime}}, \\
& H_{3}(u)=\varepsilon_{3} \sum_{\alpha=1}^{n} r_{\alpha} \frac{\sigma\left(\omega+\omega^{\prime}+U-R_{\alpha}\right)}{\sigma\left(\omega+\omega^{\prime}-R_{\alpha}\right) \sigma(U)} e^{\left(-U \eta-U \eta^{\prime}\right)} .
\end{aligned}
$$

Эллиптические решения уравнений ассоциативности соответствуют функции Бейкера-Ахиезера, определенной формулой (5.9) с $l=3$ и $R_{1}=0, R_{2}=z_{0}$, $R_{3}=-z_{0}$. Приведем соответствуюшие формулы для простейшей функции Бейкера-Ахиезера

$$
\psi(u, z)=\frac{\sigma(z+s)}{\sigma(z) \sigma(s)} e^{\Omega(u, z)} .
$$

Коэффициенты разложений

$$
\begin{gathered}
\psi=1 / z+x^{1}(u)+y^{1}(u) z+O\left(z^{2}\right) \\
\psi=x^{2}+y^{2}(u)\left(z-z_{0}\right)+O\left(\left(z-z_{0}\right)^{2}\right), \quad \psi=x^{3}+y^{3}(u)\left(z+z_{0}\right)+O\left(\left(z+z_{0}\right)^{2}\right)
\end{gathered}
$$

определяют решение

$$
F=x^{1} y^{1}-\frac{1}{2}\left(x^{2} y^{3}+x^{3} y^{2}\right)
$$

уравнений ассоциативности. Имеем

$$
\begin{gathered}
x^{1}=\zeta(U)-\wp(\omega) u^{1}-\wp\left(\omega^{\prime}\right) u^{2}-\wp\left(\omega+\omega^{\prime}\right) u^{3}, \\
x^{2}=\frac{\sigma\left(z_{0}+U\right)}{\sigma\left(z_{0}\right) \sigma(U)} \exp \Omega\left(u, z_{0}\right), \quad x^{3}=\frac{\sigma\left(U-z_{0}\right)}{\sigma\left(-z_{0}\right) \sigma(U)} \exp \Omega\left(u,-z_{0}\right)
\end{gathered}
$$

и

$$
\begin{aligned}
& y^{1}=\frac{\sigma^{\prime \prime}(U)}{2 \sigma(U)}-\zeta(U) \sum_{i=1}^{3}\left(\wp\left(\omega_{i}\right) u^{i}\right)+\frac{1}{2}\left(\sum_{i=1}^{3} \wp\left(\omega_{i}\right) u^{i}\right)^{2}, \\
& y^{2}=x^{2}(u)\left(\zeta\left(z_{0}+U\right)-\zeta\left(z_{0}\right)-\sum_{i=1}^{3} \wp\left(z_{0}-\omega_{i}\right) u^{i}\right), \\
& y^{3}=x^{3}(u)\left(\zeta\left(-z_{0}+U\right)+\zeta\left(z_{0}\right)-\sum_{i=1}^{3} \wp\left(z_{0}-\omega_{i}\right) u^{i}\right) .
\end{aligned}
$$

Функция $\widehat{F}=F-\frac{1}{2}\left(x^{1}\right)^{2}$ имеет те же третьи производные, что и функщия $F$. Следовательно, после подстановки выражений для $x^{i}$ и $y^{i}$ в (5.10) мы получим формулу для простейшего эллиптического решения уравнений ассоциативности

$$
\widehat{F}=-\frac{1}{2} \wp(U)-\frac{1}{2}\left(\wp(U)-\wp\left(z_{0}\right)\right)\left(\zeta\left(z_{0}-U\right)-\zeta\left(z_{0}+U\right)-\sum_{i=1}^{3} \wp\left(z_{0}-\omega_{i}\right) u^{i}\right) .
$$


В заключение автор выражает признательность В. Е. Захарову и С. В. Манакову за плодотворные обсуждения их замечательных работ, послуживших отправной точкой настоящего исследования.

\section{ЛитеРАтУРА}

1. Darboux G. Lecons sur le systems ortogonaux et les coordones curvilignes. Paris, 1910.

2. Дубровин Б. А., Новиков С. П. Гамильтонов формализм одномерных систем гидродинамического типа и метод усреднения Боголюбова-Уизема. ДАН CCCP, 27, 665-654 (1983).

3. Дубровин Б. А., Новиков С. П. Гидродинамика слабо деформированных солитонных решеток: Дифференциальная геометрия и гамильтонова теория. УМН, 44, 35-124 (1989).

4. Царев С. П. Геометрия гамильтоновых систем гидродинамического типа. Обобщенный метод годографа. Изв. АН СССР, сер. матем., 54, №5, 1048-1068 (1990).

5. Dubrovin B. Integrable systems in topological field theory. Nuclear Phys. B, 379, 627-689 (1992).

6. Krichever I. Tau-function of the universal Whitham hierarchy and topological field theories. Comm. Pure Appl. Math., 47, 1-40 (1994).

7. Zakharov $V$. Description of the $n$-ortogonal curvilinear coordinate systems and hamiltonian integrable systems of hydrodynamic type. Part 1 . Integration of the Lamé equations. Preprint (to appear in Duke Math. J.).

8. Захаров В. Е., Манаков С. В. Частное сообщение.

9. Witten $E$. The structure of the topological phase of two-dimensional gravity. Nuclear Phys. B, 340, 281-310 (1990).

10. Verlinder E., Verlinder $H$. A solution of two-dimensional topological quantim gravity. Preprint IASSNS-HEP 90/40, PUPT-1176, 1990.

11. Кричевер И. М. Алгебро-геометрическая конструкция уравнений ЗахароваШабата и их периодических решений. ДАН СССР, 227, №2, 291-294 (1976).

12. Кричевер И. М. Интегрирование нелинейных уравнений методами алгебраической геометрии. Функц. анализ и его прил., 11, вып. 1, 15-31 (1977).

13. Итс А. Р., Матвеев В. Б. Об одном классе решений уравнения КдФ. В сб. «Проблемы математической физики», 8, ЛГУ, 1976.

14. Krichever I., Babelon O., Billey E., Talon M. Spin generalization of the CalogeroMoser system and the matrix KP equation. Am. Math. Soc. Transl. (2), 170, 83-119 (1995).

15. Веселов А. П., Новиков С. П. Конечнозонные двумерные периодические операторы Шрёдингера: явные формулы и эволюционные уравнения. ДАН СССР, 279, №1, 20-24 (1984).

16. Кричевер И. М. Алгебро-геометрические двумерные операторы с самосогласованными потенциалами. Функц. анализ и его прил., 28, вып. 1, 26-40 (1994).

17. Дубровин Б. А., Кричевер И. М., Новиков С. П. Уравнение Шрёдингера в магнитном поле и римановы поверхности. ДАН СССР, 229, №1, 15-18 (1976).

Институт теоретической физики им. Л. Д. Ландау и факультет математики Columbia University
Поступило в редакцию 23 декабря 1996 г. 